\title{
Pediatric fibromyalgia
}

\author{
D. Buskila ${ }^{1}$, J. Ablin ${ }^{2}$ \\ 'Soroka Medical Center, Beer, Sheva, Israel; \\ ${ }^{2}$ Tel Aviv Sourasky Medical Center, Israel
}

\section{SUMMARY}

Fibromyalgia (FM) is currently defined as chronic widespread pain (CWP) with allodynia or hyperalgesia to pressure pain. It is classified as one of the large group of soft-tissue pain syndromes. Pain is the cardinal symptom of FM; however, most patients also experience additional symptoms such as debilitating fatigue, disrupted or non-restorative sleep, functional bowel disturbances, and a variety of neuropsychiatric problems, including cognitive dysfunction, anxiety and depressive symptoms. Its pathogenesis is not entirely understood, although it is currently believed to be the result of a central nervous system (CNS) malfunction that increases pain transmission and perception. FMS usually involves females, and in these patients it often makes its first appearance during menopause. But it is often diagnosed both in young as well as elderly individuals. Pediatric FMS is a frustrating condition affecting children and adolescents at a crucial stage of their physical and emotional development. Pediatric FMS is an important differential diagnosis to be considered in the evaluation of children suffering from widespread musculoskeletal pain, and must be differentiated from a spectrum of inflammatory joint disorders such as juvenile idiopathic arthritis (JIA), juvenile ankylosing spondylitis, etc. The management of pediatric FMS is centered on the issues of education, behavioral and cognitive change (with a strong emphasis on physical exercise), and a relatively minor role for pharmacological treatment with medications such as muscle relaxants, analgesics and tricyclic agents.

Key words: Pediatric FMS, treatment, females, education.

Reumatismo, 2012; 64 (4): 230-237

\section{INTRODUCTION}

1 ibromyalgia syndrome (FMS), the prototypical central sensitization disorder, is characterized by chronic widespread pain and fatigue. The condition is currently understood to represent the manifestation of pain amplification within the central nervous system (1). It is relatively common in the general population, with estimates of prevalence ranging between 2-4\% in various populations studied (2-4). Over the last decades, awareness of the existence of FMS as a syndrome with characteristic clinical, epidemiological and pathogenetic characteristics has gradually increased over both among the medical profession and the general population. This has undoubtedly also led to earlier and more accurate diagnosis and management.

Corresponding author: Dr. Dan Buskila

Soroka Medical Center Beer - Sheva, Israe

E-mail: dbuskila@bgumail.bgu.ac.il
FMS is considered to be prevalent in a wide range of age groups. Among female patients, it often makes its first appearance during menopause (5), but it is often di- agnosed both in young as well as elderly individuals. This paper aims to review the existing knowledge regarding the clinical and epidemiological characteristics of pediatric FMS. FMS has some distinct features in this group of patients and interacts with other conditions that are important to recognize in pediatric populations, such as the hypermobility spectrum.

\section{Epidemiology}

Nearly two decades ago, Buskila et al. used manual dolorimetry to evaluate the prevalence of FMS in a group of 338 Israeli children, aged 9 to 15 years (6). In this sample, $6.2 \%$ were found to have FMS, with boys exhibiting less tenderness than girls. This estimate is considerably higher than that observed in most adult populations. Interestingly, however, studies of other pediatric populations have demonstrated big differences in the prevalence of FMS to that of the Israeli population; for example, $1.2 \%$ in a cohort of Mexican children (7), $1.8 \%$ 
in a population of Finish children (8). Even within the Israeli population, significantly different rates of tenderness have been documented among children of variable ethnic background (9). Studying the true prevalence of FMS among children has been hampered to some extent by the lack of clarity and unanimity regarding the most appropriate criteria for classification and diagnosis. Two sets of classification criteria have been used: those of Yunus from 1985 (10) and the more commonly used 1990 ACR FMS criteria (11).

The criteria proposed by Yunus in 1985 required fewer tender points for the diagnosis of FMS in a child than in an adult (5 instead of 11). They also incorporate additional symptoms representing the broader spectrum of FMS, as opposed to the ACR 1990 criteria that are based solely on the presence of pain and tenderness. These symptoms include anxiety, fatigue, poor sleep, headaches, irritable bowel syndrome, subjective soft tissue swelling, numbness, pain modulation by physical activity, pain modulation by weather, and pain modulation by anxiety and distress; diagnosis of FMS in a child depends on presentation of 3 of these 10 symptoms. Despite their generally broad application, it is worth noting that the ACR FMS criteria have never been validated in the pediatric population, a caveat that may considerably limit their methodological validity. Indeed, there is some evidence that application of the $4 \mathrm{~kg}$ cut off when assessing tenderness by dolorimetry is not applicable in children, and that a $3 \mathrm{~kg}$ cut off would, in fact, be more appropriate (12).

The age distribution of FMS in the pediatric population has not been clearly defined. Reports by Eraso et al. indicate that in a considerable proportion of patients the manifestations may appear before the age of ten years and may be under-recognized (13). In another clinical report of 59 children diagnosed with FMS at a pediatric rheumatology clinic, the mean age of symptom onset was 13.7 years and the mean age of diagnosis was 15.5 years (14). Similar to FMS in adults, pediatric FMS appears to be considerably more common in females than males, although precise data regarding the size of this difference is not available. Thus, 41 of $45(91 \%)$ patients in our study were female (15).

\section{Etiology}

When considering the etiology of pediatric FMS, or of FMS in general, it is important to draw a clear distinction between pathogenesis (i.e. pathological processes involved in the propagation, amplification and preservation of increased pain, about which we have gathered considerable knowledge over the last couple of decades) and an understanding of the original cause (the Greek aitia) which still needs to be clarified. For the moment, it is generally accepted that FMS is the result of the combination of a genetic predisposition and the effect of various extrinsic stressors that results in a process of so-called central sensitization of the central nervous system (CNS). In other words, in this condition, the CNS is seen to overreact to the input of a variety of stimuli, ranging from experimental pressure applied to the thumb (16), to naturally occurring sensory stimuli such as smell or noise (17), in fact, overlapping and coinciding with over-reactivity to input originating in internal cues, such as the degree of stretch of the gastrointestinal tract (18). This paper offers a brief review of the nature of both genetic predisposition and of the major external stressors considered to be involved in causing FMS in children.

\section{Genetic predisposition}

Familial aggregation is a well-documented feature of FMS in general $(19,20)$ and the extent of this association is so great that a genetic basis for the disorder is generally assumed. This has naturally led to efforts to clarify the genetic underpinnings. The candidate gene approach was the first to be examined and a variety of target genetic markers have, therefore, been investigated. Given the role attributed to various neurotransmitters in pain processing, candidate gene studies have looked at genetic markers related to serotoninergic, dopaminergic and noradrenergic transmission in the FMS population at large (21-23). A 
gene associated with a dorsal root ganglia sodium channelopathy has also been described in a group of Mexican patients with severe FMS (24).

\section{The role of hypermobility}

Hypermobility syndrome, which is also usually considered a genetic condition, is a well know clinical association of FMS in children (25).

In one study of 338 children (mean age 11.5 years), the prevalence of hypermobility was $13 \%$ and the prevalence of FMS was $6 \%$, with a considerable overlap between the two conditions; $81 \%$ of the children diagnosed with FMS met the criteria for hypermobility (26). The precise nature of this association is not, however, absolutely clear. It may seem an attractive and intuitive proposition to believe that hyperlaxity may lead to recurrent micro trauma and occasional joint dislocation, thus acting as a basis for recurrent peripheral pain eventually leading to the development of central sensitization and FMS. It appears, however, that additional factors may be involved. Dysautonomia is a common associated factor in the hypermobility syndrome, and symptoms such as postural tachycardia, orthostatic intolerance and orthostatic hypotension are common in this condition (27). Pain and distress also appear to interact in this population of patients (28). As pointed out in a recent review, the joint hypermobility syndrome is currently perceived as a multisystem and under-diagnosed heritable disorder of connective tissue, in which chronic pain is one of the most difficult clinical manifestations (29).

\section{Disordered sleep and FMS}

In a study comparing polysomnographic findings of pediatric FMS patients and their mothers, Roizenblatt et al. demonstrated a significant concordance regarding the diagnosis of FMS between the children and their mothers (30). A significant correlation was observed between polysomnographic indexes, sleep anomalies, and pain manifestations in both children and their mothers. Specific sleep disorders such as periodic limb movements in sleep
(PLMS) have been documented in pediatric FMS patients (31). In a classical report by Yunus et al. on 33 FMS patients aged 17 years or under (10), sleep disturbances were reported in $67 \%$ of cases. Intriguingly, while sleep disruption may initially be directly related to the level of pain, these symptoms may eventually develop into a separate, primary sleep disorder due to other behavioral and psychosocial factors (32). It, therefore, appears that, like the situation in adults, sleep disorders play a significant role in the etiology of pediatric FMS as well.

Growing pains are the most common pain syndrome of childhood, estimated to affect up to $10-20 \%$ of children aged 3-12 years $(33,34)$. Children suffering from growing pains have been reported to have an increased number of tender points and decreased pain thresholds (35), indicating that this condition may be a forerunner for the development of other chronic pain conditions, including FMS. Recent research indicates that growing pain is associated with a widespread disorder of somatosensory processing (36).

Another fascinating aspect of chronic pain in children relates to the possibility of early life exposure to pain priming the central nervous system and resulting in enhanced pain processing at a later stage of life. Evidence for this hypothesis has indicated that, for example, neonatal circumcision can result in an increased response to the pain from vaccination events in later life (37). Similarly, surviving pre-term infants have been shown to demonstrate increased tenderness and reduced pain thresholds later in life, although they do not exhibit clinical pain (38). The mechanism through which early life experience may influence subsequent pain susceptibility is not completely understood, but it is possible that activation (programming) of the autonomic nervous system and of the hypothalamic-pituitary axis may be involved (39).

It has been suggested that stress and distress play an important role as triggers for the development of FMS later on in childhood (as well as in adulthood). Stressors such as single parent families, histories of 
sexual abuse, and learning difficulties have been reported to be common among children with idiopathic musculoskeletal pain (40). A range of childhood adversities have been reported among children suffering from FMS, including not only sexual abuse but also factors such as a poor emotional relationship with parents, lack of physical affection, experiences of parents' physically violent quarrels, alcohol or other problems of addiction in the mother, separation, and domestic financial difficulties before the age of 7 years (41). Increased distress and chronic pain are evident in families of adolescents with FMS (42), and adolescents with FMS have been shown to experience problems in peer relationships (43). A recent systematic review that attempted to evaluate the evidence connecting FMS with a history of childhood abuse (emotional, physical and sexual) concluded that the reported evidence supports the association but the quality of the studies is relatively low. Also low study quality tended to be associated with higher effect sizes for sexual abuse in childhood (44).

\section{Clinical presentation}

While the intrinsic hallmark of pediatric FMS remains by definition the presence of chronic widespread pain in a child, similar to the situation with adults, it is becoming increasingly apparent that the spectrum of FMS is, in fact, much broader than originally conceived, encompassing a wide array of symptoms not directly related to pain and tenderness. Fatigue is a major part of the FMS spectrum in children and can be a highly debilitating symptom, interfering with educational as well as social functioning (45). Children who suffer from FMS also appear to score high on scales of depression, even when compared with children who suffer from chronic pain but do not meet the criteria of FMS (8). In a large clinical report describing the clinical manifestations of pediatric FMS patients, Gedalia et al. reported diffuse aching in $97 \%$, headaches in $76 \%$, sleep disturbances in $69 \%$, stiffness in $29 \%$, subjective joint swelling in $24 \%$, fatigue in $20 \%$, and abdominal pain in $17 \%$ (14). Joint hyper- mobility was reported in $14 \%$ of children. Another case series reported higher rates of sleep disturbances and fatigue ( $>90 \%$ and $62 \%$, respectively), a figure that is more similar to adult rates (15). The overlap between FMS and chronic fatigue in children has been recognized for a long time and Bell et al. reported that $30 \%$ of children diagnosed with chronic fatigue actually met criteria for FMS (46). Currently, since both chronic fatigue and FMS are considered to be part of the same spectrum of functional disorders characterized by central nervous system sensitization $(47,48)$, this association seems obvious and, in fact, it would not be surprising to find that the overlap may be even larger.

For the moment, the optimal set of criteria for the diagnosis of pediatric FMS has still not been agreed on. Using tender points as a measurement of biological trait of tenderness is known to be highly subjective (49) and, therefore, in adults, the ACR is in the process of developing new diagnostic criteria for FMS which will eliminate the use of tender $(50,51)$ points while incorporating the spectrum of associated symptoms of FMS, not addressed by the original 1990 ACR criteria (11). It is not clear, however, whether these developments will be applicable to children and specific work is called for in order to update the diagnosis of FMS in children.

\section{Treatment}

The management of FMS at any age can broadly be divided into pharmacological and the non-pharmacological. FMS is a syndrome of a specific biological derangement; enhanced pain processing within the central nervous system leads to a series of negative results gradually exerting a greater general effect on the quality of life and level of function of the individual involved. Therefore, maladaptive cognitive and behavioral patterns are a compounding factor (52); a reduction in the level of physical activity, social interaction, educational achievement, and the development of anxiety and depression are all well known spin offs of the original pain disorder. Understanding this downward spiral is essential 
in order to adequately tackle the various aspects of the fibromyalgia syndrome. The attending physician must simultaneously attempt to reverse the negative behavioral patterns while choosing specific pharmacological interventions that target the underlying pain disorder. At the same time, it is crucial to identify (or to rule out) underlying and alternative generators of pain, ranging from local pathologies (traumatic, developmental, etc.) to systemic inflammatory (or other) disorders.

Education is crucial for early intervention in the management of pediatric FMS. Both the child and the parents need to be informed about the nature of the disorder, expectations should be coordinated and anxieties addressed. Stressing the non-progressive nature of FMS is important and helpful. Specific issues that may be causing anxiety should be actively sought after and managed. Therefore, in some cases, a teenage female patient or her parents may be concerned about the future impact of FMS on her fertility and the course of pregnancy. In other cases, the possibility of other children developing similar symptoms may be of concern to the parents. These issues should be discussed openly. Often, explaining the nature of the disorder actually comes as a relief for the patient and the family, making a hitherto confusing situation shrouded in mystery more comprehensible and manageable.

\section{Exercise}

A randomized controlled trial of a 12-week exercise intervention in children with FMS demonstrated significant improvements in physical function, functional capacity, quality of life and fatigue in the children treated with aerobic exercise of moderate intensity (53). Exercise has been shown to be effective over a range of pediatric rheumatological disorders, including FMS juvenile idiopathic arthritis (JIA), juvenile systemic lupus erythematosus (SLE) and juvenile dermatomyositis (54).

\section{Cognitive behavioral treatment (CBT)}

Since cognitive centers of the central nervous system play an important role in the processing of pain (55), it is natural to assume that cognitive interventions may act as modulators of the pain response and can have a positive effect on the underlying pathological basis of FMS. In adults, this has, in fact, been substantiated. Not only is cognitive behavioral therapy an evidencebased treatment modality in FMS (56), but functional imaging studies have demonstrated that specific anatomical areas of the brain involved in the cognitive processing of pain can actually alter their response following a CBT intervention (57).

Functional brain imaging has made it possible to assess the effect of cognitive factors such as catastrophizing on pain processing (58). Catastrophizing is associated with increased activity in brain areas related to pain anticipation (medial frontal cortex, cerebellum), pain-attention (dorsal anterior cingulate gyrus, dorsolateral prefrontal cortex), and emotional aspects of pain (claustrum, closely connected to the amygdala).

CBT-based techniques, such as cognitive restructuring, thought stopping, distraction, relaxation, and self-reward have been effectively been used in pediatric FMS (59). A large meta-analysis conducted by Palermo et al. has concluded that psychological therapies result in improvement in pain relief across several different pain conditions in children (60), and a recent Cochrane review has concluded that psychological treatments may improve pain control for children with a variety of pain conditions, including muscle pain, abdominal pain, headaches and FMS (61). Therefore, it appears that CBT should be offered as a preferred modality of non-pharmacological treatment for pediatric FMS.

\section{Pharmacological treatment}

Surprisingly, little evidence is available regarding the pharmacological treatment of FMS in children. While three medications, including two serotonin-norepinephrine reuptake inhibitors (duloxetine and milnacipran) and one alpha-2-delta ligand (pregabalin) have been approved by the US Food and Drug Administration (FDA) for the treatment of FMS in adults (62), 
none of these medications have been tested in well-controlled trials in pediatric FMS. Furthermore, black box warnings are in place for duloxetine and milnacipran (as well as for amitryptiline) regarding the increased risk of suicide in children treated with these medications. While this lack of evidence confirms the need for future research to develop specific pharmacological treatment for pediatric FMS, it is apparent that, for the moment, the management of FMS in children must rely heavily rely on the implementation of non-pharmacological interventions, as described above.

\section{Prognosis}

Buskila et al. have carried out a prospective study of outcome of FMS in children over 30 months of follow up (63). In this study, a high proportion of children (73\%) appeared not to fulfill FMS criteria at the end of the follow-up period; the number of tender points also decreased significantly. These results implied that the outcome of FMS among children may, in fact, be more favorable than that in adults, among whom FMS tends to behave as a chronic disorder in most cases. Siegel et al. also reported that improvement had been seen in most children with FMS after a mean of 2.6 years after presentation (15).

\section{CONCLUSIONS}

Pediatric FMS is a frustrating condition affecting children and adolescents at a crucial stage of their physical and emotional development. Although it does not lead to deformity or mortality, the condition causes considerable impairment of quality of life, disrupts social and educational achievements, and is a cause for anxiety and concern for patients and their parents. Despite this, the condition appears to carry a better outcome when compared with that in adults. Pediatric FMS is an important differential diagnosis to be considered in the evaluation of children suffering from widespread musculoskeletal pain, and must be differentiated from a spectrum of inflammatory joint disorders such as JIA, juvenile ankylosing spondylitis, etc. The management of pediatric FMS is centered on the issues of education, behavioral and cognitive change (with a strong emphasis on physical exercise), and a relatively minor role for pharmacological treatment with medications such as muscle relaxants, analgesics and tricyclic agents. Further research is called for in order to evaluate both the specific pathophysiology of FMS in children as well as the applicability of advanced pharmacological treatment in this population.

\section{REFERENCES}

1. Phillips K, Clauw DJ. Central pain mechanisms in chronic pain states-maybe it is all in their head. Best Pract Res Clin Rheumatol. 2011; 25: 141-54.

2. Mas AJ, Carmona L, Valverde M, Ribas B. Prevalence and impact of fibromyalgia on function and quality of life in individuals from the general population: results from a nationwide study in Spain. Clin Exp Rheumatol. 2008; 26: 519-26.

3. Branco JC, Bannwarth B, Failde I, et al. Prevalence of fibromyalgia: a survey in five European countries. Semin Arthritis Rheum. 2010; 39: 448-53.

4. Wolfe F, Ross K, Anderson J, et al. The prevalence and characteristics of fibromyalgia in the general population. Arthritis Rheum. 1995; 38: 19-28.

5. Waxman J, Zatzkis SM. Fibromyalgia and menopause. Examination of the relationship. Postgrad Med. 1986; 80: 165-1.

6. Buskila D, Press J, Gedalia A, et al. Assessment of nonarticular tenderness and prevalence of fibromyalgia in children. J Rheumatology. 1993; 20: 368-70.

7. Clark P, Burgos-Vargas R, Medina-Palma C, et al. Prevalence of fibromyalgia in children: a clinical study of Mexican children. J Rheumatol. 1998; 25: 2009-14.

8. Mikkelsson M, Sourander A, Piha J, Salminen JJ. Psychiatric symptoms in preadolescents with musculoskeletal pain and fibromyalgia. Pediatrics. 1997; 100: 220-7.

9. Buskila D, Neumann L, Press J, et al. Assessment of nonarticular tenderness of children in different ethnic groups. J Musculoskeletal Pain. 1995; 3: 83-90.

10. Yunus MB, Masi AT. Juvenile primary fibromyalgia syndrome. A clinical study of thirtythree patients and matched normal controls. Arthritis Rheum. 1985; 28: 138-45.

11. Wolfe F, Smythe HA, Yunus MB, et al. The 
American College of Rheumatology 1990 Criteria for the Classification of Fibromyalgia. Report of the Multicenter Criteria Committee. Arthritis \& Rheumatism. 1990; 33: 160-72.

12. Neumann L, Smythe HA, Buskila D. Performance of point count and dolorimetry in assessing nonarticular tenderness in children. J Musculoskeletal Pain. 1996; 4: 29-35.

13. Eraso RM, Bradford NJ, Fontenot CN, Espinoza LR, Gedalia A. Fibromyalgia syndrome in young children: onset at age 10 years and younger. Clin Exp Rheumatol. 2007; 25: 63944.

14. Gedalia A, Garcia CO, Molina JF, Bradford NJ, Espinoza LR. Fibromyalgia syndrome: experience in a pediatric rheumatology clinic. Clin Exp Rheumatol. 2000; 18: 415-9.

15. Siegel DM, Janeway D, Baum J. Fibromyalgia syndrome in children and adolescents: clinical features at presentation and status at followup. Pediatrics. 1998; 101: 377-82.

16. Pujol J, Lopez-Sola M, Ortiz H, Vilanova JC, Harrison BJ, Yucel M, et al. Mapping brain response to pain in fibromyalgia patients using temporal analysis of FMRI. PLoS One. 2009; 4: e5224.

17. Wilbarger JL, Cook DB. Multisensory hypersensitivity in women with fibromyalgia: implications for well being and intervention. Arch Phys Med Rehabil. 2011; 92 (4): 653-6.

18. Wilder-Smith CH, Robert-Yap J. Abnormal endogenous pain modulation and somatic and visceral hypersensitivity in female patients with irritable bowel syndrome. World J Gastroenterol. 2007; 13: 3699-704.

19. Buskila D, Neumann L, Hazanov I, Carmi R. Familial aggregation in the fibromyalgia syndrome. Semin Arthritis Rheum. 1996; 26: 605-11.

20. Arnold LM, Hudson JI, Hess EV, et al. Family study of fibromyalgia. Arthritis Rheum. 2004; 50: 944-52.

21. Cohen H, Buskila D, Neumann L, Ebstein RP. Confirmation of an association between fibromyalgia and serotonin transporter promoter region (5- HTTLPR) polymorphism, and relationship to anxiety-related personality traits. Arthritis Rheum. 2002; 46: 845-7.

22. Buskila D, Cohen H, Neumann L, Ebstein RP. An association between fibromyalgia and the dopamine D4 receptor exon III repeat polymorphism and relationship to novelty seeking personality traits. Mol Psychiatry. 2004; 9: 730-1.

23. Vargas-Alarcon G, Fragoso JM, Cruz-Robles $\mathrm{D}$, et al. Catechol-O-methyltransferase gene haplotypes in Mexican and Spanish patients with fibromyalgia. Arthritis Res Ther. 2007; 9: R110.

24. Vargas-Alarcon G, Alvarez-Leon E, Fragoso JM, et al. A SCN9A gene-encoded dorsal root ganglia sodium channel polymorphism associ- ated with severe fibromyalgia. BMC Musculoskelet Disord. 2012; 13: 23.

25. Sendur OF, Gurer G, Bozbas GT. The frequency of hypermobility and its relationship with clinical findings of fibromyalgia patients. Clin Rheumatol 2007; 26: 485-7.

26. Gedalia A, Press J, Klein M, Buskila D. Joint hypermobility and fibromyalgia in schoolchildren. Ann Rheum Dis. 1993; 52: 494-6.

27. Gazit Y, Nahir AM, Grahame R, Jacob G. Dysautonomia in the joint hypermobility syndrome. Am J Med. 2003; 115: 33-40.

28. Grahame R. Pain, distress and joint hyperlaxity. Joint Bone Spine. 2000; 67: 157-63.

29. Grahame R. Joint hypermobility syndrome pain. Curr Pain Headache Rep. 2009; 13: $427-$ 33.

30. Roizenblatt S, Tufik S, Goldenberg J, et al. Juvenile fibromyalgia: clinical and polysomnographic aspects. J Rheumatol. 1997; 24 : 579-85.

31. Tayag-Kier CE, Keenan GF, Scalzi LV, et al. Sleep and periodic limb movement in sleep in juvenile fibromyalgia. Pediatrics. 2000; 106 : E70.

32. Palermo TM, Wilson AC, Lewandowski AS, et al. Behavioral and psychosocial factors associated with insomnia in adolescents with chronic pain. Pain. 2011; 152: 89-94.

33. Uziel Y, Chapnick G, Jaber L, et al. Five-year outcome of children with "growing pains": correlations with pain threshold. J Pediatr. 2010; 156: 838-40.

34. Uziel Y, Hashkes PJ. Growing pains in children. Pediatr Rheumatol Online J. 2007; 5: 5.

35. Hashkes PJ, Friedland O, Jaber L, et al. Decreased pain threshold in children with growing pains. J Rheumatol. 2004; 31: 610-3.

36. Pathirana S, Champion D, Jaaniste T, et al. Somatosensory test responses in children with growing pains. J Pain Res. 2011; 4: 393-400.

37. Taddio A, Katz J, Ilersich AL, Koren G. Effect of neonatal circumcision on pain response during subsequent routine vaccination. Lancet. 1997; 349: 599-603.

38. Buskila D, Neumann L, Zmora E, et al. Pain sensitivity in prematurely born adolescents. Arch Pediatr Adolesc Med. 2003; 157 (11): 1079-82.

39. Phillips DI, Jones A. Fetal programming of autonomic and HPA function: do people who were small babies have enhanced stress responses? J Physiol. 2006; 572: 45-50.

40. Malleson PN, al-Matar M, Petty RE. Idiopathic musculoskeletal pain syndromes in children. J Rheumatol. 1992; 19: 1786-9.

41. Imbierowicz K, Egle UT. Childhood adversities in patients with fibromyalgia and somatoform pain disorder. Eur J Pain. 2003; 7: 113-9.

42. Kashikar-Zuck S, Lynch AM, Slater S, et al. Family factors, emotional functioning, and functional impairment in juvenile fibromy- 
algia syndrome. Arthritis Rheum. 2008; 59: 1392-8.

43. Kashikar-Zuck S, Lynch AM, Graham TB, et al.Social functioning and peer relationships of adolescents with juvenile fibromyalgia syndrome. Arthritis Rheum. 2007; 57: 47480.

44. Hauser W, Kosseva M, Uceyler N, et al. Emotional, physical, and sexual abuse in fibromyalgia syndrome: a systematic review with meta-analysis. Arthritis Care Res (Hoboken). 2011; 63: 808-20.

45. Varni JW, Burwinkle TM, Limbers CA, Szer IS. The PedsQL as a patient-reported outcome in children and adolescents with fibromyalgia: an analysis of OMERACT domains. Health Qual Life Outcomes. 2007; 5: 9.

46. Bell DS, Bell KM, Cheney PR. Primary juvenile fibromyalgia syndrome and chronic fatigue syndrome in adolescents. Clin Infect Dis. 1994; 18: S21-S3.

47. Yunus MB. Role of central sensitization in symptoms beyond muscle pain, and the evaluation of a patient with widespread pain. Best Pract Res Clin Rheumatol. 2007; 21: 481-97.

48. Yunus MB. Central sensitivity syndromes: a new paradigm and group nosology for fibromyalgia and overlapping conditions, and the related issue of disease versus illness. Semin Arthritis Rheum. 2008; 37: 339-52.

49. Petzke F, Gracely RH, Park KM, et al. What do tender points measure? Influence of distress on 4 measures of tenderness. J Rheumatol. 2003; 30: 567-74.

50. Wolfe F, Clauw DJ, Fitzcharles MA, et al. Fibromyalgia criteria and severity scales for clinical and epidemiological studies: a modification of the ACR Preliminary Diagnostic Criteria for Fibromyalgia. J Rheumatol. 2011; 38: 1113-22.

51. Wolfe F, Clauw DJ, Fitzcharles MA, et al. The American College of Rheumatology preliminary diagnostic criteria for fibromyalgia and measurement of symptom severity. Arthritis Care Res (Hoboken). 2010; 62: 600-10.

52. Ablin JN, Cohen H, Neumann L, et al. Coping styles in fibromyalgia: effect of co-morbid posttraumatic stress disorder. Rheumatol Int. 2008; 28: 649-56.

53. Stephens S, Feldman BM, Bradley N, et al. Feasibility and effectiveness of an aerobic exercise program in children with fibromyalgia: results of a randomized controlled pilot trial. Arthritis Rheum. 2008; 59: 1399-406.

54. Gualano B, Pinto AL, Perondi MB, et al. Therapeutic effects of exercise training in patients with pediatric rheumatic diseases. Rev Bras Reumatol. 2011; 51: 490-6.

55. Villemure C, Bushnell MC. Cognitive modulation of pain: how do attention and emotion influence pain processing? Pain. 2002; 95: 195-9.

56. Williams DA, Cary MA, Groner KH, et al. Improving physical functional status in patients with fibromyalgia: a brief cognitive behavioral intervention. J Rheumatol. 2002; 29: 1280-6.

57. Farrell MJ, Van Meter JW, Petzke F, et al. Supraspinal activity associated with painful pressure in fibromyalgia is associated with beliefs about locus of pain control. Arthritis Reum. 2001; 44: S394-9.

58. Gracely RH, Geisser ME, Giesecke T, et al. Pain catastrophizing and neural responses to pain among persons with fibromyalgia. Brain. 2004; 127: 835-43.

59. Degotardi PJ, Klass ES, Rosenberg BS, et al. Development and evaluation of a cognitivebehavioral intervention for juvenile fibromyalgia. J Pediatr Psychol 2006; 31: 714-23.

60. Palermo TM, Eccleston C, Lewandowski AS, et al. Randomized controlled trials of psychological therapies for management of chronic pain in children and adolescents: an updated meta-analytic review. Pain. 2010; 148: 387-97.

61. Eccleston C, Palermo TM, Williams AC, et al. Psychological therapies for the management of chronic and recurrent pain in children and adolescents. Cochrane Database Syst Rev 2009; CD003968.

62. Crofford LJ. Pain management in fibromyalgia. Curr Opin Rheumatol. 2008; 20: 246-50.

63. Buskila D, Neumann L, Hershman E, et al. Fibromyalgia syndrome in children-an outcome study. J Rheumatol. 1995; 22: 525-8. 